\title{
Calibration of a Polarimetric Microwave Radiometer Using a Double Directional Coupler
}

\author{
Luisa de la Fuente $^{1, *(\mathbb{D}}$, Beatriz Aja ${ }^{1} \mathbb{D}$, Enrique Villa $^{2}(\mathbb{D})$ and Eduardo Artal $^{1}$ \\ 1 Departamento de Ingeniería de Comunicaciones, Universidad de Cantabria, 39005 Santander, Spain; \\ ajab@unican.es (B.A.); artale@unican.es (E.A.) \\ 2 IACTEC, Instituto de Astrofísica de Canarias, 38205 La Laguna, Spain; evilla@iac.es \\ * Correspondence: luisa.delafuente@unican.es
}

Citation: de la Fuente, L.; Aja, B.; Villa, E.; Artal, E. Calibration of a Polarimetric Microwave Radiometer Using a Double Directional Coupler. Remote Sens. 2021, 13, 2109

https://doi.org/10.3390/rs13112109

Academic Editor: Isaac Ramos

Received: 19 April 2021

Accepted: 26 May 2021

Published: 27 May 2021

Publisher's Note: MDPI stays neutral with regard to jurisdictional claims in published maps and institutional affiliations.

Copyright: (c) 2021 by the authors. Licensee MDPI, Basel, Switzerland. This article is an open access article distributed under the terms and conditions of the Creative Commons Attribution (CC BY) license (https:// creativecommons.org/licenses/by/ $4.0 /)$.
Abstract: This paper presents a built-in calibration procedure of a 10 -to- $20 \mathrm{GHz}$ polarimeter aimed at measuring the I, Q, U Stokes parameters of cosmic microwave background (CMB) radiation. A full-band square waveguide double directional coupler, mounted in the antenna-feed system, is used to inject differently polarized reference waves. A brief description of the polarimetric microwave radiometer and the system calibration injector is also reported. A fully polarimetric calibration is also possible using the designed double directional coupler, although the presented calibration method in this paper is proposed to obtain three of the four Stokes parameters with the introduced microwave receiver, since $\mathrm{V}$ parameter is expected to be zero for the $\mathrm{CMB}$ radiation. Experimental results are presented for linearly polarized input waves in order to validate the built-in calibration system.

Keywords: radiometer; polarimeter calibration; microwave polarimeter; radiometer calibration; radio astronomy receiver; cosmic microwave background receiver; Stokes parameters

\section{Introduction}

Remote sensing applications employ sensitive instruments to fulfill the scientific goals of dedicated missions or projects, either space or terrestrial. The involved instruments can be divided into two primary types: passive and active sensors [1]. They basically differ in the manner of measuring the radiation from the object. In a passive sensor, the natural radiation emitted from the object or scene being observed is detected. On the other hand, active sensors illuminate the object under observation and the instrument measures the reflected radiation from the object. Typical remote sensing applications cover a wide range of the electromagnetic spectrum from $380 \mathrm{~nm}$ to $1 \mathrm{~m}$ wavelengths, including the visible light up to $780 \mathrm{~nm}$, infrared (from $780 \mathrm{~nm}$ to $0.1 \mathrm{~mm}$ ), terahertz and microwave ranges [1].

Particularly, microwave technology emerged in the early 1960s providing a different view and additional information from remote sensing [2]. Microwaves penetrate clouds and more deeply into vegetation compared to optical waves, attributing improved sensing capabilities independently of weather conditions to microwave systems. Microwave passive instruments are known as radiometers, whereas active ones as radars [1]. Among the last ones, different classes are found, such as synthetic-aperture radar (SAR) systems [3-5], side-looking airborne radar (SLAR), scatterometers [6], altimeters and meteorological radars applied for diverse applications. Contrastingly, passive microwave radiometry (PMR) has been involved in moisture measurements or meteorological applications $[7,8]$ among others. However, PMR in remote sensing can be applied not only to spaceborne radiometers, but also to terrestrial experiments. These ones are based on radio telescopes and focused on sky observation to characterize different cosmological parameters depending on the application. Among these parameters, the measurement of the cosmic microwave background $(\mathrm{CMB})$ and the definition of its features, particularly its anisotropies and polarization, are common scientific goals of recent instruments [9-12]. 
Therefore, detecting the polarization of astronomical waves coming from the sky provides valuable scientific information for a better knowledge of the universe, its origin and its expected evolution. The CMB is a relic radiation which has attracted a sustained interest in the cosmology science community for decades, and it is still under observation both in terrestrial and space radio astronomy observatories, with a large number of big experiments currently running and also with new ones under development to operate in a near future. The $\mathrm{CMB}$ radiation pattern corresponds to linearly polarized waves, in a small percentage $[13,14]$. A good knowledge of this polarization could demonstrate the existence of gravitational waves in the early universe, through an accurate measurement of primordial B-modes [15].

CMB received signals are very weak, as typically occurs in radio astronomy applications, and the use of very high sensitivity receivers is mandatory. Noise equivalent temperature of such receivers must be as low as possible to reduce observation time and to provide accurate recorded data. Several topologies have been typically employed in the design of $\mathrm{CMB}$ experiments, such as a Dicke receiver adapted with polarization modulators or correlation schemes [16]. Nevertheless, for the most recent CMB polarization experiments a polarimetric radiometer scheme is the preferred choice [17-21]. The front-end in a polarimetric receiver is necessarily composed of devices able to separate orthogonal components of the incoming wave. Some kind of internal correlators provide output signals proportional to combinations of the Stokes parameters, which are the most useful magnitudes to determine the polarization degree of an electromagnetic wave [22]. Since CMB radiation has a certain degree of linear polarization and it is free of circularly polarized components, some CMB polarimetric receivers are designed to obtain three Stokes parameters: I, Q and $\mathrm{U}$, because $\mathrm{V}$ parameter is expected to be zero. Some ground-based experiments have detected a weakly circularly polarized radiation from atmospheric molecular oxygen, considered as a contaminant [23]. However, the polarized atmospheric $\mathrm{O}_{2}$ signal decreases significantly below $20 \mathrm{GHz}$ [23].

Calibration of polarimetric radiometers is a critical issue, since the observed polarization data depend on accurate measurements of polar components. Some polarimeter CMB receivers use an external sky source for calibration $[15,18,24]$. However, the inaccuracy of such polarization calibrators is then directly translated to the Stokes parameters. On the other hand, several methods employing external calibrators are commonly used, enabling the injection of several waves with different polarizations. The linearly polarized signals can be obtained by means of a wire grid [25] or rotatable dielectric sheets [26], while a dielectric retardation plate is added for a circular polarization signal for a fully polarimetric calibration $[27,28]$. From a different perspective, other calibration methods apply the injection of polarized test waves through components such as directional couplers [29-32], or coaxial probes inserted in a circular waveguide [33]. On the other hand, polarimetric active radar calibrators have been demonstrated for radar polarimeter calibration [34].

This paper presents a built-in calibrator system based on the injection of signals through a broadband double directional coupler (DDC) $[35,36]$ for a polarimetric radiometer working in 10 to $14 \mathrm{GHz}$ and 16 to $20 \mathrm{GHz}$ bands. Any arbitrary polarized wave can be synthesized by an adequate combination of the amplitude and the phase of the injected signals in two separated input ports of the DDC. When calibration signals are off, the DDC does not affect the sky signal received by the radiometer. The system can be calibrated in short time periods selected by the user. Experimental tests in a laboratory test-bench have demonstrated the proper operation of the proposed calibration system, for different synthesized polarized waves.

\section{Polarimeter Description}

The polarimeter presented in this paper is aimed at measuring the Stokes parameters of a polarized incident wave covering two frequency ranges from 10 to $14 \mathrm{GHz}$ and from 16 to $20 \mathrm{GHz}$. The ground-based experiment is expected to operate at Teide Observatory 
(2390 m, a.s.l, Canary Islands, Spain). In the following subsections the architecture of the polarimeter as well as the calibration injector are described.

\subsection{Receiver Architecture}

The block diagram of the full receiver is shown in Figure 1. The receiver provides four output voltage signals for each band. Each output signal is proportional to a linear combination of the three Stokes parameters (I, Q, U). In general, those parameters, in terms of the two linear orthogonal electric field components, in a standard Cartesian basis $(x, y)$, are expressed as time averages of electric field components shown in the Stokes vector (1):

$$
\left[\begin{array}{c}
I \\
Q \\
U \\
V
\end{array}\right]=\left[\begin{array}{c}
\left\langle E_{x}^{2}\right\rangle+\left\langle E_{y}^{2}\right\rangle \\
\left\langle E_{x}^{2}\right\rangle-\left\langle E_{y}^{2}\right\rangle \\
2 \cdot \operatorname{Re}\left\langle E_{x} E_{y}^{*}\right\rangle \\
-2 \cdot \operatorname{Im}\left\langle E_{x} E_{y}^{*}\right\rangle
\end{array}\right]
$$

where the two linear orthogonal electric fields $E_{x}$ and $E_{y}$, are the components of the total electric field in rectangular (Cartesian) coordinates which is expressed as the combination of horizontal (x) and vertical (y) field components, with unitary vectors $\hat{x}$ and $\hat{y}$ as:

$$
\bar{E}=E_{x} \hat{x}+E_{y} \hat{y}
$$

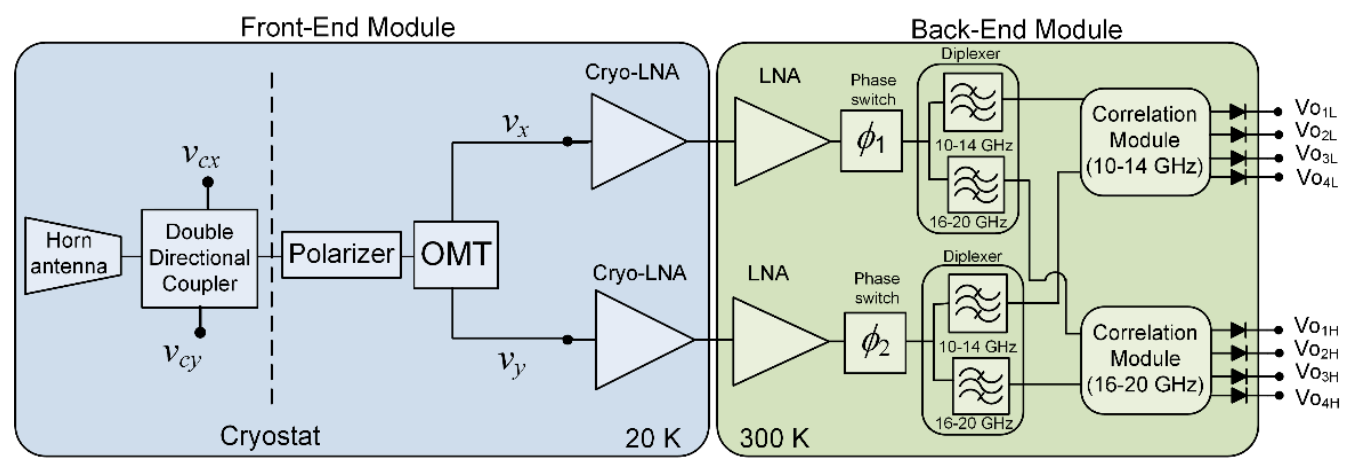

Figure 1. Block diagram of the full receiver, with the front-end module (FEM) operating at cryogenic temperature (in blue) and the back-end module (BEM) at ambient temperature (in green).

The whole receiver comprises a front-end module (FEM) cooled down to $20 \mathrm{~K}$ and a back-end module (BEM) at $300 \mathrm{~K}$ [37]. As the FEM operates at cryogenic temperature, the equivalent noise temperature of the receiver is reduced to a few dozens of Kelvin.

In the FEM, the received radiation wave, after passing through the horn antenna, the double directional coupler (DDC) and the polarizer, is split into two components through an ortho-mode transducer (OMT). The $(x, y)$ axis orientation of the incident field is defined by the OMT, whereas the polarizer is rotated $45^{\circ}$ with relation to the $x$ axis of the OMT (see Appendix A for details). The OMT outputs, through waveguide to coaxial adapters, become the voltages $v_{x}$ and $v_{y}$, which are complex magnitudes or phasors, with frequency dependent amplitude and phase values. In terms of the two orthogonal electric fields $E_{x}$ and $E_{y}$, those voltages are given by:

$$
\begin{gathered}
v_{x}=\frac{1}{\sqrt{2}}\left(E_{x}-j E_{y}\right) \\
v_{y}=\frac{1}{\sqrt{2}}\left(-j E_{x}+E_{y}\right)
\end{gathered}
$$


Two cryogenic low-noise amplifiers (LNA) are placed just at the OMT outputs to reduce the system noise temperature. Then, these voltages are amplified and filtered through two branches in the BEM and correlated in a correlation module, in order to obtain four outputs to calculate the Stokes parameters simultaneously. The amplifier stage in the BEM adjusts the signal level required for the output detectors without increasing the thermal load in the cryogenic stage and with a negligible contribution to the system noise. The use of a phase-switching module in both branches enables a variable phase modulation between the two polarization components. The small discrete phase step $\left(5.625^{\circ}\right)$ provided by the two 6-bit digital phase shifters (phase switches in the block diagram), leads to redundant information, and a more accurate correction of systematic errors is expected. The filtering stage splits the full frequency band into two sub-bands, $10-14 \mathrm{GHz}$ and $16-20 \mathrm{GHz}$, thus avoiding the interfering signal at $15 \mathrm{GHz}$ present at the observatory location. The operation principle of the correlation module is described in Appendix B. Microwave signals at correlation module outputs, prior to quadratic law detection, considering ideal subsystems and amplifiers with unit gain, are:

$$
\begin{aligned}
& v_{1}=\frac{-1}{2 \sqrt{2}}\left[j E_{x}\left(1-e^{j \phi}\right)+E_{y}\left(1+e^{j \phi}\right)\right] \\
& v_{2}=\frac{-1}{2 \sqrt{2}}\left[E_{x}\left(1+e^{j \phi}\right)+j E_{y}\left(e^{j \phi}-1\right)\right] \\
& v_{3}=\frac{-1}{2 \sqrt{2}}\left[E_{x}\left(1+j e^{j \phi}\right)-E_{y}\left(j+e^{j \phi}\right)\right] \\
& v_{4}=\frac{-1}{2 \sqrt{2}}\left[E_{x}\left(1-j e^{j \phi}\right)+E_{y}\left(e^{j \phi}-j\right)\right]
\end{aligned}
$$

where $\phi=\phi_{2}-\phi_{1}$ is the phase difference between branches.

In the next expressions, the orthogonal electric field values, $E_{x}$ and $E_{y}$, are assumed to be real magnitudes, and the quadratic diode detectors are supposed to be ideal microwave to video converters, providing output voltages (DC Volt) according to:

$$
V_{o k}=\left|v_{k}\right|^{2} \text { with } k=1,2,3,4
$$

Correlation module output video voltages, $V_{o k}$, expressed in terms of the electric field components, are then given by:

$$
\left[\begin{array}{l}
V_{o 1} \\
V_{o 2} \\
V_{o 3} \\
V_{o 4}
\end{array}\right]=\frac{1}{4}\left[\begin{array}{ccc}
(1-\cos \phi) & (1+\cos \phi) & \sin \phi \\
(1+\cos \phi) & (1-\cos \phi) & \sin \phi \\
(1-\sin \phi) & (1+\sin \phi) & -\cos \phi \\
(1+\sin \phi) & (1-\sin \phi) & \cos \phi
\end{array}\right]\left[\begin{array}{c}
E_{x}^{2} \\
E_{y}^{2} \\
2 E_{x} E_{y}
\end{array}\right]
$$

In terms of Stokes parameters, these output voltages, $V_{o k}$, for each receiver frequency band, can be expressed as:

$$
\left[\begin{array}{l}
V_{o 1} \\
V_{o 2} \\
V_{o 3} \\
V_{o 4}
\end{array}\right]=\frac{1}{4}\left[\begin{array}{ccc}
1 & -\cos \phi & \sin \phi \\
1 & \cos \phi & \sin \phi \\
1 & -\sin \phi & -\cos \phi \\
1 & \sin \phi & \cos \phi
\end{array}\right]\left[\begin{array}{c}
I \\
Q \\
U
\end{array}\right]
$$

Since the final application of the instrument is to measure the polarization of the CMB, which contents only linearly polarized waves, the Stokes parameter V is expected to be zero. The type of correlation module used in the present receiver is not designed to measure the Stokes parameter V, but its design is addressed to measure the three Stokes parameters I, Q and $U$. 


\subsection{Calibration Injector}

For calibration purposes, a broadband double directional coupler (DDC) $[35,36]$ based on a pair of rectangular waveguide directional couplers is mounted in the FEM of the receiver, just behind the antenna. Its insertion loss can be considered constant since it is thermally stabilized. This coupler allows injecting a set of calibration waves composed of linearly polarized waves for a polarimetric calibration to measure I, Q and U Stokes parameters. The DDC configuration also allows the synthesis of an arbitrary elliptically polarized wave as incoming radiation for a fully polarimetric radiometer calibration with a receiver suitable to measure the four Stokes parameters. Since during the normal operation of the polarimeter the DDC is cooled down to cryogenic temperatures, its contribution to the system noise temperature is negligible due to its very low insertion loss. A schematic description of the assembly to apply the calibration signals is depicted in Figure 2. By means of a power divider, the broadband noise source injects the noise calibration waves to the single pole double throw (SPDT) switches, which connect either the noise source, with a hot temperature $\left(T_{h}\right)$ or the cold reference load, with a cold temperature $\left(T_{c}\right)$, to the coupled ports of the DDC. With combinations of both SPDT positions, three linearly polarized waves can be injected for a zero-phase shift, (i.e., $\varphi_{1}=\varphi_{2}$ ), while a $90^{\circ}$ phase difference would be needed for a circularly polarized wave. A coupling factor in the DDC of around $32 \mathrm{~dB}$ minimizes the noise power level coming from the reference loads when the SPDT is connected to them. Moreover, it should be considered that the noise source hot temperature during the calibration process, should be chosen high enough in order to distinguish the calibration signals from the input one (the received signal coming from the horn antenna). Figure 3. shows a detail of the antenna-feed system composed of the feedhorn, the DDC, the polarizer and the OMT, whereas a detailed view of a BEM board, including subsystems of both branches and the Correlation Modules for both frequency bands, is shown in Figure 4.

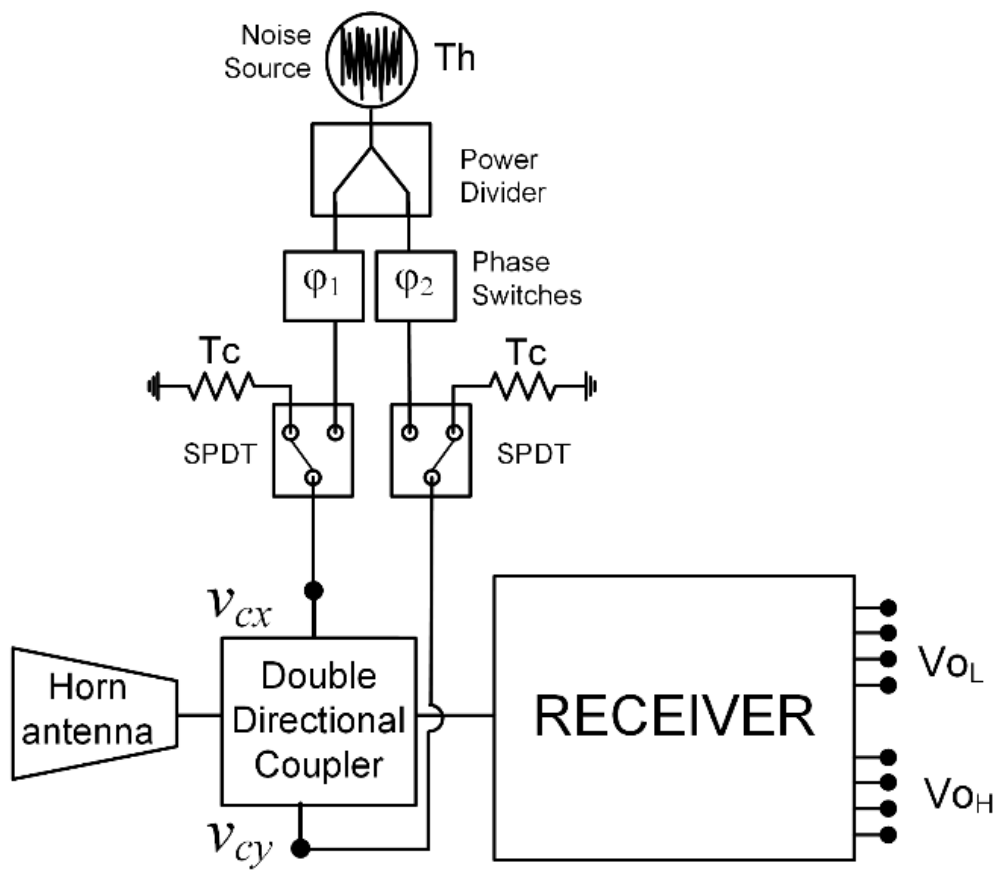

Figure 2. Schematic description of the signal calibration injector. 


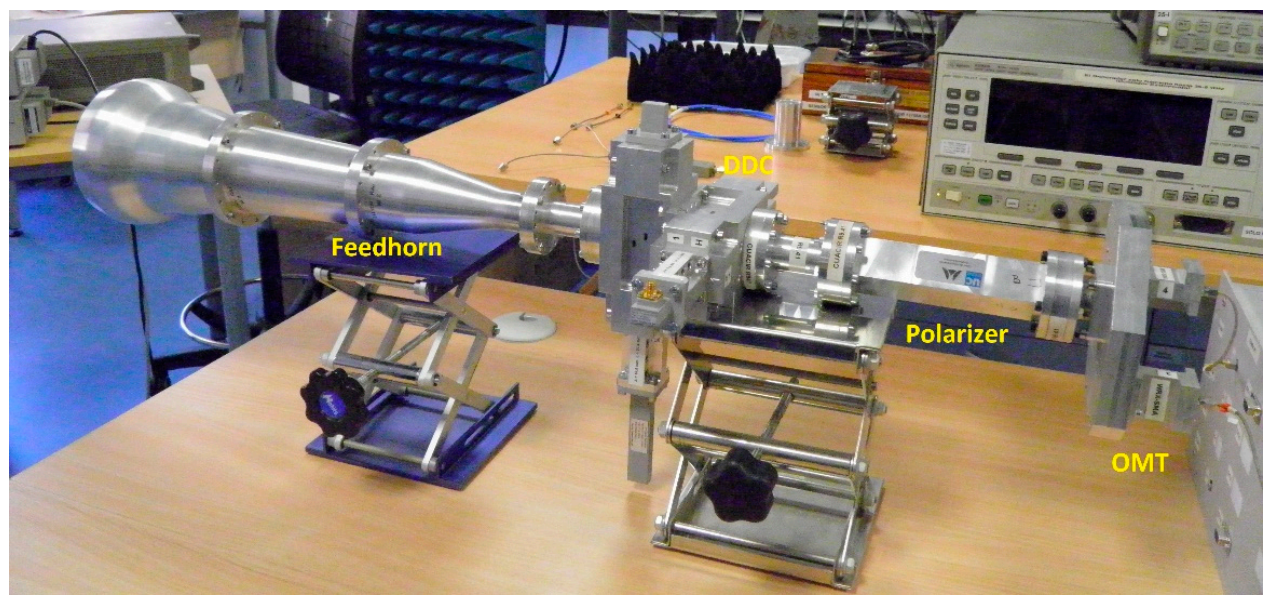

Figure 3. Detail of the antenna-feed system. From left to right: feedhorn, double directional coupler, polarizer and ortho-mode transducer.

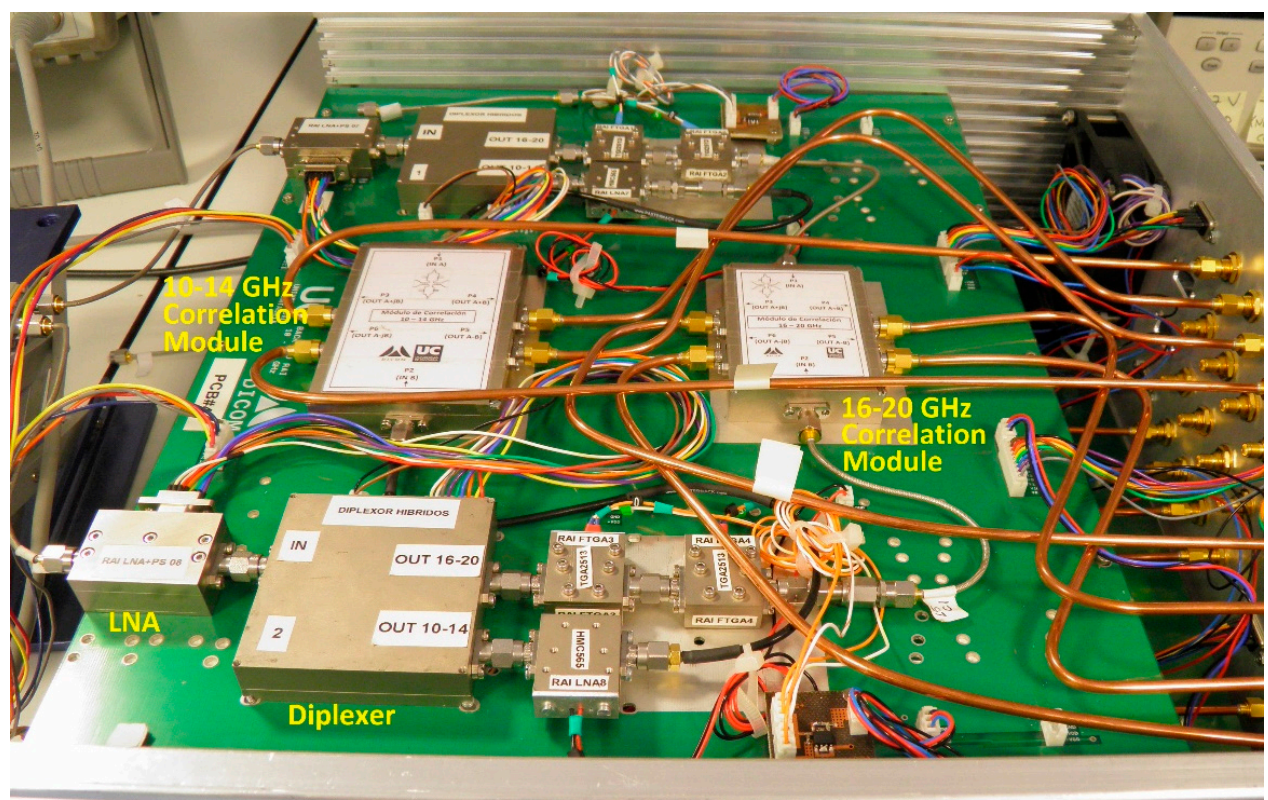

Figure 4. Back-end-module board showing all the subsystems involved for the operation at both frequency bands and the interconnections between them.

\section{Calibration Method}

Calibration procedure consists in determining the sensitivities and offset parameters of the radiometer, which contain the imbalances caused by hardware differences between branches and in the correlation modules. The four output voltages are linearly related to the first three Stokes parameters of an incident wave. Using a matrix formulation to simplify equations, the $(4 \times 1)$ matrix composed by those four video output voltages can be expressed as an output voltage vector $\overline{v_{0}}$. It is related to the Stokes parameters vector $(3 \times 1)$ $\overline{S_{i n}}$, the $(4 \times 3)$ radiometer sensitivity matrix $\overline{\bar{C}}$, and the offset voltage vector $(4 \times 1) \bar{o}$. All elements in those matrixes and vectors are real numbers. Then the forward model of the polarimetric radiometer can be written as:

$$
\overline{v_{o}}=\overline{\bar{C}} \cdot \overline{S_{i n}}+\bar{o}=\left[\begin{array}{l}
V_{o 1} \\
V_{o 2} \\
V_{o 3} \\
V_{o 4}
\end{array}\right]=\left[\begin{array}{lll}
\alpha_{11} & \alpha_{12} & \alpha_{13} \\
\alpha_{21} & \alpha_{22} & \alpha_{23} \\
\alpha_{31} & \alpha_{32} & \alpha_{33} \\
\alpha_{41} & \alpha_{42} & \alpha_{43}
\end{array}\right]\left[\begin{array}{c}
I \\
Q \\
U
\end{array}\right]_{i n}+\left[\begin{array}{c}
o_{1} \\
o_{3} \\
o_{3} \\
o_{4}
\end{array}\right]
$$


where $\overline{v_{0}}$ is the video output voltage vector, $\overline{\bar{C}}$ is the radiometer sensitivity matrix $(\mathrm{V} / \mathrm{W}), \bar{o}$ is the offset voltage vector and $\overline{S_{i n}}$ is the Stokes parameters vector of the input noise-like wave. During the calibration process, the unknown parameters, that need to be determined, are the radiometer sensitivity matrix and the offset voltage vector. Since this polarimetric radiometer exhibits sensitivity to three of the four Stokes parameters (I, Q and U), three input stimuli are required for its complete calibration.

The radiometer offset $\bar{o}$ is not related to the input calibration waves, but directly to the receiver noise temperature. Then, the output voltages measured for the cold reference loads $\left(\mathrm{T}_{\mathrm{C}}\right)$ connected to the DDC inputs, correspond to the offset parameters, obtaining an offset voltage vector for each frequency band. Moreover, through the DDC, noise powers from the noise source hot temperature $\left(T_{h}\right)$ are injected, whereas a microwave absorber is placed in front of the horn antenna for the laboratory measurements. The injected noise powers are the calibration standards for three linear polarized signals, which Stokes parameters are modelled as vectors of the input noise-like signal. The injected powers through the DDC provide received waves according to the reference coordinate axis system $(x, y)$. Those noise powers are chosen to be above the noise of the receiver to get a high enough noticeable video output voltages with relation to the offset voltages. Moreover, the injected powers should be low enough to ensure the linear operation of the receiver, without gain compression issues. The received power spectral densities (PSD) of horizontal $(x)$ or vertical $(y)$ wave components at polarizer input ports are given by:

$$
P S D_{x, y}(W / H z)=\frac{k T_{h}}{L_{P w D} L_{S P D T} C_{x, y}}
$$

where $T_{h}$ is the hot noise temperature of the noise source, $L_{P w D}$ is the insertion loss of the power divider, $L_{S P D T}$ is the insertion loss of the SPDT and $C_{x, y}$ is the coupling factor for each input of the DDC. Moreover, the phase difference between injected noise powers in each reference axis is considered during the calibration, $\varphi_{x, y}=\varphi_{2}-\varphi_{1}$ (see Figure 2). The effective bandwidth $B$, of each frequency band, defines the horizontal $(x)$ or vertical component $(y)$ power expressed as:

$$
P_{x, y}(W)=P S D_{x, y} \cdot B
$$

The normalized spectral response for both frequency bands, $10-14 \mathrm{GHz}$ and 16-20 GHz, is depicted in Figure 5, for each one of the four detected outputs. In both bands the effective bandwidth is around $4.1 \mathrm{GHz}$.

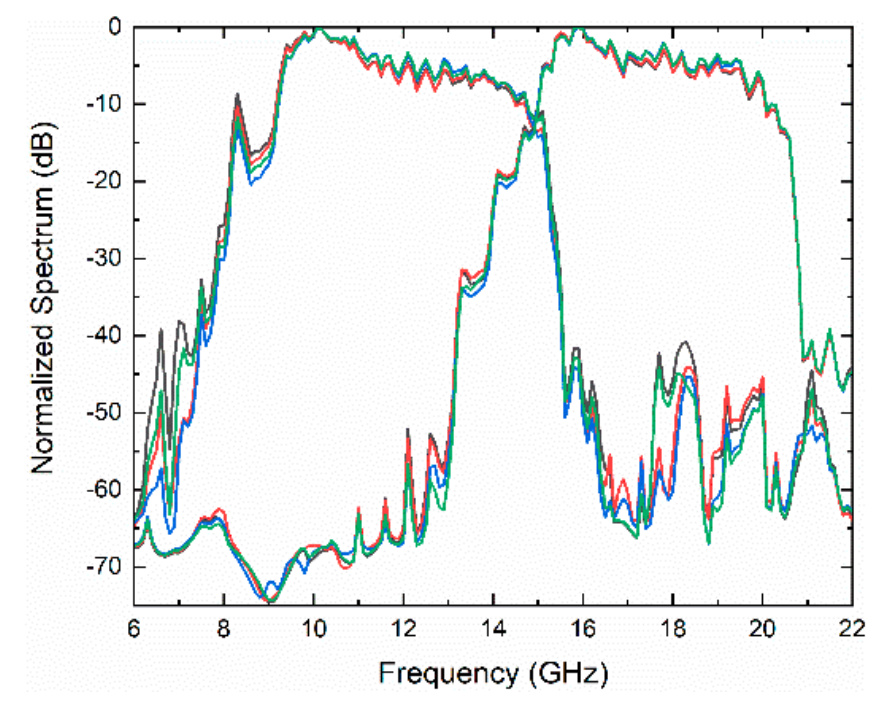

Figure 5. Normalized spectral response of the receiver of the four detected outputs for both frequency bands. 
Therefore, the Stokes parameters of the injected signal can be expressed as a combination of horizontal and vertical power components as:

$$
\overline{S_{i n}}=\left[\begin{array}{c}
I \\
Q \\
U
\end{array}\right]_{i n}=\left[\begin{array}{c}
P_{x}+P_{y} \\
P_{x}-P_{y} \\
2 \sqrt{P_{x}} \sqrt{P_{y}} \cos \left(\varphi_{x, y}\right)
\end{array}\right]
$$

With the aim of calibrating the instrument, three types of linearly polarized waves are injected: horizontal, vertical and $45^{\circ}$. These waves are obtained by switching the SPDTs in Figure 2. between the noise source $\left(T_{h}\right)$ and the cold reference load $\left(T_{c}\right)$, with the settings listed in Table 1.

Table 1. Injected polarized waves for calibration.

\begin{tabular}{|c|c|c|c|c|}
\hline Wave & $\begin{array}{l}\text { Position SPDT } \\
\left(v_{c x}\right)\end{array}$ & $\begin{array}{c}\text { Position SPDT } \\
\left(v_{c y}\right)\end{array}$ & $\begin{array}{c}\text { Injected Wave Phase } \\
\varphi_{x, y}=\varphi_{2}-\varphi_{1}\end{array}$ & $\begin{array}{c}\text { Stokes Power Vector } \\
\frac{S_{i n p}}{}\end{array}$ \\
\hline $\begin{array}{l}\text { Linearly Horizontal polarized } \\
\left(0^{\circ}\right) ; p=H\end{array}$ & Noise source $\left(T_{h}\right)$ & Reference load $\left(\mathrm{T}_{\mathrm{c}}\right)$ & $0^{\circ}$ & {$\left[\begin{array}{c}P_{x} \\
P_{x} \\
0\end{array}\right]$} \\
\hline $\begin{array}{l}\text { Linearly Vertical polarized } \\
\left(90^{\circ}\right) ; p=V\end{array}$ & Reference load $\left(T_{c}\right)$ & Noise source $\left(T_{h}\right)$ & $0^{\circ}$ & {$\left[\begin{array}{c}P_{y} \\
-P_{y} \\
0\end{array}\right]$} \\
\hline $\begin{array}{l}\text { Linearly } 45^{\circ} \text { polarized } \\
\left(45^{\circ}\right) ; p=45\end{array}$ & Noise source $\left(T_{h}\right)$ & Noise source $\left(T_{h}\right)$ & $0^{\circ}$ & $\begin{array}{c}P_{x}+P_{y} \\
P_{x}-P_{y} \\
2 \sqrt{P_{x}} \sqrt{P_{y}} \cos \left(\varphi_{x, y}\right)\end{array}$ \\
\hline
\end{tabular}

The offset is subtracted from the receiver output voltages for each one of the linearly polarized coupled waves. Expressing the Stokes parameters of the injected signal in terms of input power, the detected voltages can be defined as:

$$
\overline{v_{p}}=\overline{v_{o p}}-\bar{o}=\overline{\bar{C}} \cdot \overline{S_{i n p}}
$$

with $p=H, V, 45^{\circ}$.

The twelve elements of the radiometer sensitivity matrix can be obtained as:

$$
\begin{gathered}
\alpha_{k 1}=\frac{1}{2}\left(\frac{v_{H}(k)}{P_{x}}+\frac{v_{V}(k)}{P_{y}}\right) \\
\alpha_{k 2}=\frac{1}{2}\left(\frac{v_{H}(k)}{P_{x}}-\frac{v_{V}(k)}{P_{y}}\right) \\
\alpha_{k 3}=\frac{v_{45}(k)-\alpha_{k 1}\left(P_{x}+P_{y}\right)-\alpha_{k 2}\left(P_{x}-P_{y}\right)}{2 \sqrt{P_{x}} \sqrt{P_{y}} \cos \left(\varphi_{x, y}\right)}
\end{gathered}
$$

where $k=1,2,3,4$ correspond to each one of the four BEM output voltages. Equations (16) to (19) are solved for each frequency band, obtaining a radiometer sensitivity matrix for each one.

After calibrating the radiometer, the Stokes parameters vector of any unknown input wave can be computed numerically using a pseudo-inverse matrix according to:

$$
\overline{S_{u}}=\left(\overline{\bar{C}}^{T} \cdot \overline{\bar{C}}\right)^{-1} \cdot \overline{\bar{C}}^{T} \cdot\left(\overline{v_{o}}-\bar{o}\right)
$$

where $\overline{v_{0}}$ is the measured output voltages vector for any arbitrary unknown input wave $[18,27,29]$. 


\section{Experimental Results}

In this section the presented instrument has been calibrated following the proposed method, in order to measure the Stokes parameters of an unknown wave. The measurements are made indoor, with the polarimeter working at ambient temperature $(300 \mathrm{~K})$ as a proof of concept.

\subsection{Polarimetric Radiometer Calibration}

The hot temperature of the noise source, for applying the calibration standards, has been adjusted considering the actual system noise temperature, the receiver dynamic range and the coupling factor of the DDC. The receiver noise temperature is around $225 \mathrm{~K}$. The linearly polarized waves required for the calibration have been obtained by switching the SPDTs between the reference cold load and the noise source (see Figure 2.). The detected voltages are measured with a data acquisition system with $0.5 \mathrm{~s}$ integration time. The radiometer sensitivity matrix $\overline{\bar{C}}(\mathrm{~V} / \mathrm{W})$ and the offset voltage vector $\bar{o}(\mathrm{~V})$ are obtained for an $11.25^{\circ}$ phase step of the BEM phase switches module. The twelve sensitivity matrix coefficients $\alpha_{k j}(k=1,2,3,4 ; j=1,2,3)$ and the offset voltage vectors for the $10-14 \mathrm{GHz}$ and $16-20 \mathrm{GHz}$ bands versus the phase difference between branches in the BEM $\phi=\phi_{2}-\phi_{1}$ are shown in Figure 6. The offset voltage vector elements, $O_{k}$, due to the receiver noise temperature and to the $300 \mathrm{~K}$ background input noise, are of the order of millivolts. The coefficients $\alpha_{k 1}$ show an almost constant behavior with the phase modulation, since it represents the sensitivity to the intensity of the input wave. The coefficients $\alpha_{k 2}$ and $\alpha_{k 3}$ are the sensitivity of the radiometer to the $Q$ and $U$ Stokes parameters, and they show a dependence on the applied modulating phase as described in Equation (11) and shown in Figure 6.

Additionally, a calibration was performed switching the phase state in the BEM between $\phi=0^{\circ}$ and $\phi=180^{\circ}$, with $\phi=\phi_{2}-\phi_{1}$. In this case, a single radiometer sensitivity matrix $\overline{\bar{C}}(\mathrm{~V} / \mathrm{W})$ is obtained using the amplitude of the squared detected output voltages after removing the offset voltage vector $\bar{o}(\mathrm{~V})$. This radiometer performance removes systematic errors and avoids gain or noise temperature fluctuations slower than the switching time ( $1 \mathrm{~s})$. The sensitivity matrix coefficients for the $10-14 \mathrm{GHz}$ and $16-20 \mathrm{GHz}$ bands are:

$$
\begin{aligned}
\overline{\bar{C}}_{0,180 ; 10-14 \mathrm{GHz}} & =\left[\begin{array}{ccc}
2.331 & 50.329 & -6.343 \\
0.5423 & -47.169 & 6.191 \\
1.374 & 4.418 & 47.57 \\
1.4521 & -47.169 & -52.463
\end{array}\right](\mathrm{V} / \mu \mathrm{W}) \\
\overline{\bar{C}}_{0,180 ; 16-20 \mathrm{GHz}} & =\left[\begin{array}{ccc}
0.1339 & 42.825 & -4.192 \\
1.074 & -5.272 & 3.688 \\
1.513 & 2.325 & 24.296 \\
-0.458 & -4.316 & -28.66
\end{array}\right](\mathrm{V} / \mu \mathrm{W})
\end{aligned}
$$

An error analysis has been performed, where a matrix of estimated uncertainties has been obtained due to imperfect knowledge of the calibrator injector components. These errors can be classified into systematic or random uncertainties. There are many sources of potential systematic error. These include the uncertainties of the noise hot temperature used to calibrate, the phase switches (loss and phase), the SPDT loss and effects of radiometer passband averaging. In addition, random uncertainties include physical temperature errors. 


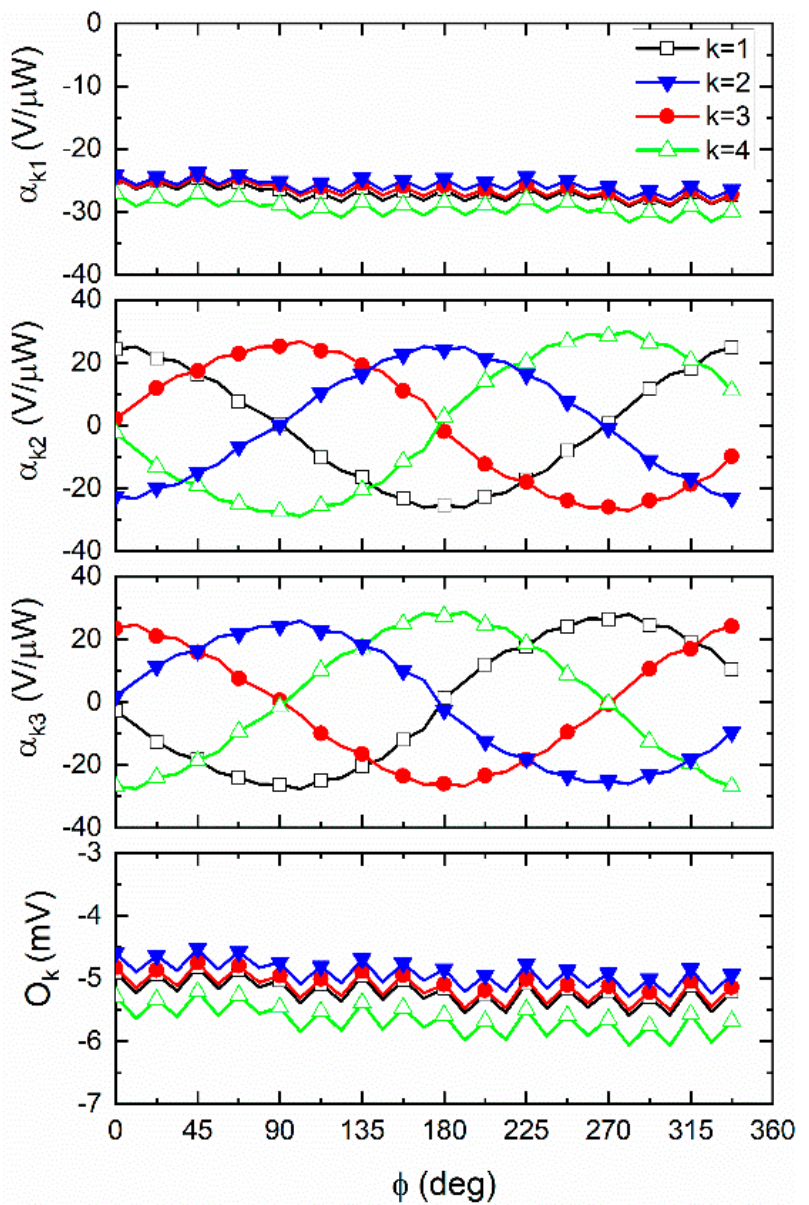

(a)

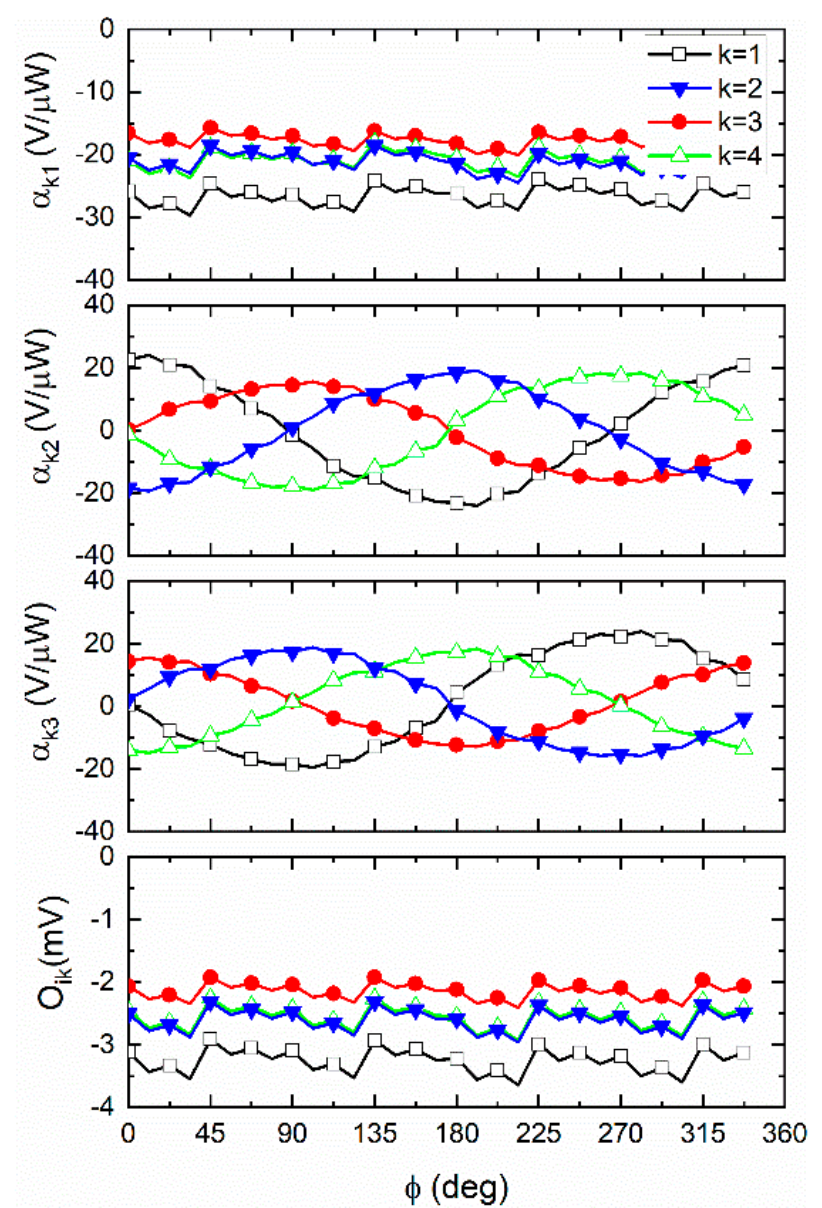

(b)

Figure 6. Results of the radiometer calibration, $\overline{\bar{C}}, \bar{o}$ in Equation (12). Sensitivity matrix coefficients, $\alpha_{k j}$, and offset voltage vector elements, $O_{k}$, with $k$ the output number, versus phase difference between branches in the BEM $\phi=\phi_{2}-\phi_{1}$. (a) 10-14 GHz frequency band, (b) 16-20 GHz frequency band.

The uncertainty sensitivity matrix $\overline{\overline{\delta C}}(\mathrm{~V} / \mathrm{W})$ is derived considering the estimated systematic and random uncertainties of the calibration standard parameters listed in Table 2.

Table 2. Estimated uncertainties for various parameters of the calibration injector.

\begin{tabular}{cccc}
\hline Calibration Standard Parameter & Noise Source ENR (dB) & Loss (dB) & Phase (deg) \\
\hline Random Uncertainty & 0.001 & 0.001 & 0.1 \\
Systematic Uncertainty & 0.22 & 0.02 & 1 \\
\hline
\end{tabular}

An uncertainty sensitivity matrix is calculated for each calibration standard parameter uncertainty. The resulting total uncertainty, estimated using standard propagation of errors for each frequency band, is dominated by the ENR (excess noise ratio) uncertainty of the noise source, and $\overline{\overline{\delta C}}$, at each frequency band, are:

$$
\overline{\overline{\delta C}}_{10-14 \mathrm{GHz}}=\left[\begin{array}{ccc}
2.03 & 1.93 & 2.09 \\
2.03 & 2.09 & 2.11 \\
2.09 & 2.07 & 2.12 \\
2.07 & 2.74 & 2.09
\end{array}\right] \%
$$




$$
\overline{\overline{\delta C}}_{16-20 \mathrm{GHz}}=\left[\begin{array}{ccc}
1.74 & 4.77 & 1.84 \\
1.72 & 1.84 & 1.84 \\
1.84 & 1.79 & 1.85 \\
1.79 & 2.63 & 1.84
\end{array}\right] \%
$$

The uncertainty due to low-frequency noise from thermal fluctuations and readout electronics is expected to be mitigated by the phase-switch modulation.

\subsection{Measurement of a Broadband Polarized Noise Wave}

A broadband polarized noise wave was measured to demonstrate the radiometer calibration method. The experiment consisted of a noise diode connected to a transmitting antenna with linear vertical polarization, i.e., polar angle of $90^{\circ}$. The transmitting antenna is a broadband log-periodic antenna with a gain of $8.5 \mathrm{~dB}, \mathrm{R} \& S \mathrm{~s}$ can be modified rotating the transmitting antenna, by using a coaxial rotary joint. Figure 7 shows the measurement test-bench in the laboratory. The polar angle of the source can be modified rotating the transmitting antenna, by using a coaxial rotary joint. Since the radiometer is working at ambient temperature, the transmitted power is chosen for a linear operation of the receiver and sufficiently above its equivalent noise temperature. The operation of the receiver is demonstrated as a total power radiometer, for a fixed phase switches state.

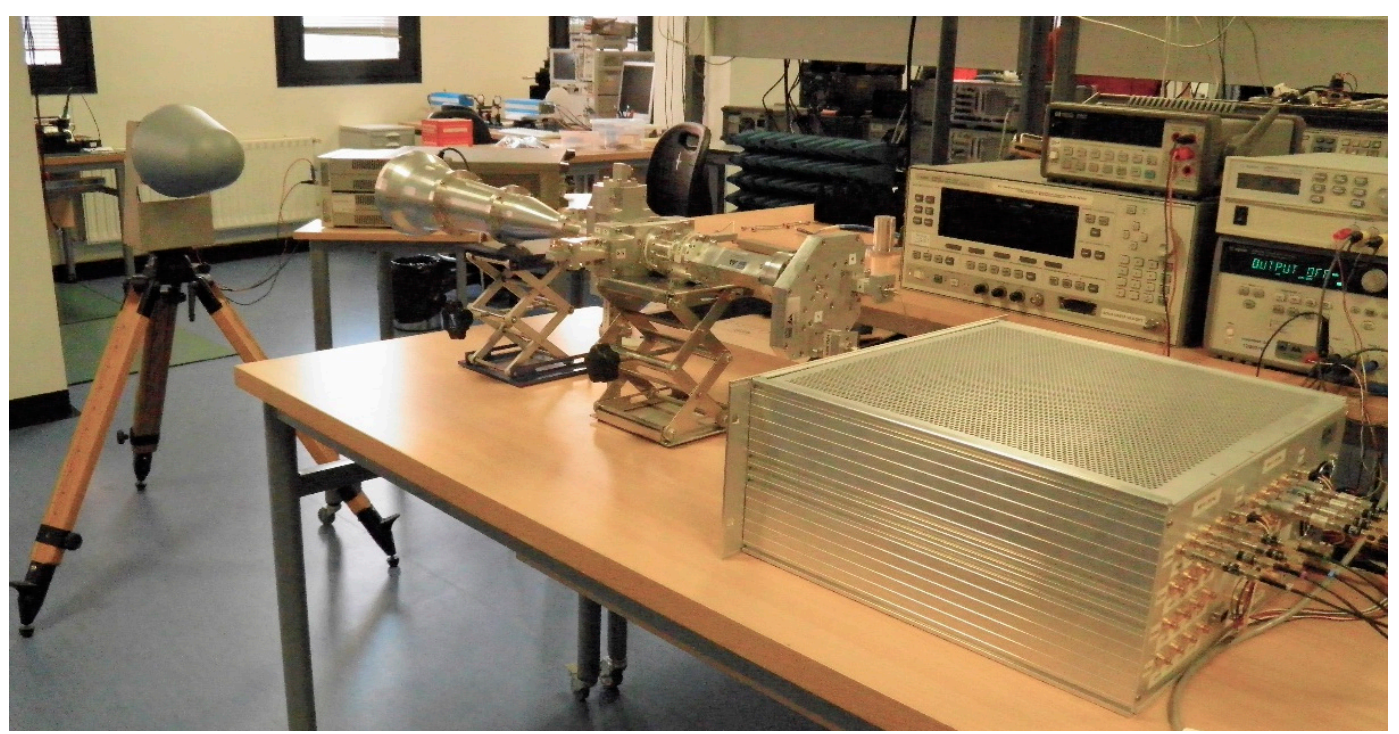

Figure 7. Radiometer measurement experimental set-up.

First, the four detected voltages of both frequency bands are acquired for a fixed phase state $\phi=0^{\circ}$. The corresponding offset voltages, obtained during the calibration process, are subtracted in order to obtain the Stokes parameters of the received wave $\overline{S_{u}}$, applying Equation (20), with the calibration sensitivity matrix of the radiometer obtained for the phase state $0^{\circ}$. The measured Stokes parameters (I, Q and U), in terms of power and normalized to the intensity for both frequency bands, are:

$$
\begin{aligned}
& \left.\overline{S_{u}}\right|_{10-14 \mathrm{GHz}}=\left[\begin{array}{c}
44.813 \\
-43.204 \\
0.445
\end{array}\right](n W) ;\left.\overline{S_{u}}\right|_{10-14 \mathrm{GHz}}=I_{i n}\left[\begin{array}{c}
1.0000 \\
-0.9641 \\
0.0099
\end{array}\right] \\
& \left.\overline{S_{u}}\right|_{16-20 \mathrm{GHz}}=\left[\begin{array}{c}
30.986 \\
-28.812 \\
-1.286
\end{array}\right](n W) ;\left.\overline{S_{u}}\right|_{16-20 \mathrm{GHz}}=I_{\text {in }}\left[\begin{array}{c}
1.0000 \\
-0.9299 \\
-0.0415
\end{array}\right]
\end{aligned}
$$

The obtained results show a polar angle of $87.85^{\circ}$ for the $10-14 \mathrm{GHz}$ frequency band and $96.47^{\circ}$ for the $16-20 \mathrm{GHz}$ frequency band, for a theoretical $90^{\circ}$ polar angle. Although 
the obtained results demonstrate the calibration method, there is a small deviation in the measured polar angle with respect to the transmitted one. A source of uncertainty in the measurement is caused by the coordinate axis alignment between the transmitting and receiving antennas, since they are placed on different base holders. Systematic errors are another source of uncertainty. Moreover, the results for the 16-20 GHz frequency band show higher systematic errors since the polarizer and the DDC operating bandwidth is limited to $19.5 \mathrm{GHz}$, but frequencies above this value are not rejected by the diplexer (see Figure 5).

\section{Discussion}

The calibration technique presented herein demonstrates the capacity to retrieve the $\mathrm{I}, \mathrm{Q}$ and $\mathrm{U}$ Stokes parameters of an incoming radiation wave, when the polarimeter is working at ambient temperature $(300 \mathrm{~K})$. The instrument with the Front-End Module (FEM) cooled down to a cryogenic temperature of $20 \mathrm{~K}$ will have an equivalent noise temperature of around $16 \mathrm{~K}$ [37], which will reduce observation time and provide accurate data from the CMB. For the laboratory measurements, the receiver is working as a total power radiometer and the transmitted signal power is set according to the receiver dynamic range, although the phase switches in the BEM can be used to modulate the incoming signal in a coherent detection in order to detect weaker signals. The switching speed should be fast enough to overcome the 1 / $\mathrm{f}$ noise when pairs of detected correlated outputs are subtracted. Because the available phase step is as low as $5.625^{\circ}$, a nearly continuous modulation is possible, entailing the cancelation of time-variant systematic errors in the receiver.

The calibration procedure using the DDC has both similar advantages and limitations when compared to other methods for calibrating polarimetric radiometers. One limitation is that the effect of the radiometer antenna is not calibrated such as cross-polarization leakage, which is not included in the terms of the polarimetric sensitivity matrix. This sensitivity matrix characterizes the receiver performance including the polarizer, orthomode transducer, cryogenic low-noise amplifiers and the BEM components. On the other hand, both the DDC and the polarizer make use of square waveguides, which limit the operational frequency bands, due to the existence of higher propagation modes. However, the advantages of the presented calibrator injector are not only the possibility to calibrate the radiometer periodically in the operating mode, but also its simple and static structure that can be cryogenically cooled. Moreover, the DDC exhibits an inherent low cross-polarization and, therefore, a modification of the incoming polar wave is prevented.

\section{Conclusions}

In this work, a built-in injector system in applied to a polarimetric radiometer calibration. The described calibration injector uses a broadband double directional coupler (DDC), which is a rectangular waveguide coupler integrated behind the feedhorn antenna. This calibration system can be used to inject linearly, circularly or elliptically polarized waves in order to achieve a fully polarimetric calibration. The proposed calibration method is applied to a polarimetric radiometer intended for measuring I, Q and U Stokes parameters of the cosmic microwave background, switching periodically a noise source to synthesize linearly polarized waves. The calibration method has been demonstrated determining the sensitivity matrix and the offset voltage vector of a polarimetric radiometer using a set of three linearly polarized waves. The calibration procedure has been validated by experimental tests with several polarized waves transmitted from a far field broadband antenna having low cross-polarization properties.

Author Contributions: Conceptualization, E.A. and B.A.; methodology, E.A., L.d.l.F., B.A. and E.V.; software, B.A. and L.d.l.F.; validation, L.d.l.F. and B.A.; formal analysis, E.A.; investigation, E.A. and E.V.; resources, B.A. and L.d.l.F.; data curation, B.A. and L.d.1.F.; writing-original draft preparation, B.A., L.d.l.F., E.V. and E.A.; writing—review and editing, B.A., L.d.l.F., E.V. and E.A.; visualization, B.A.; supervision, E.A.; project administration, E.A.; funding acquisition, E.A. All authors have read and agreed to the published version of the manuscript. 
Funding: The authors would like to thank the Spanish Ministry of Science and Innovation for financial support provided through the grants ESP2015-70646-C2-2-R, ESP2017-83921-C2-2-R and PID2019-110610RB-C22.

Institutional Review Board Statement: Not applicable.

Informed Consent Statement: Not applicable.

Data Availability Statement: Data Sharing Not applicable.

Acknowledgments: The authors would like to thank J.L. Cano, and A. Mediavilla for the design and the development of part of the subsystems. They also would like to thank E. M. Cuerno and P. García for the fabrication and assembly of passive and active subsystems.

Conflicts of Interest: The authors declare no conflict of interest. The funders had no role in the design of the study; in the collection, analyses, or interpretation of data; in the writing of the manuscript, or in the decision to publish the results.

\section{Appendix A}

First components in the FEM of the receiver are passive waveguide devices which separate the electromagnetic field components of a received wave. The feedhorn antenna with a circular waveguide output does not modify the polarization of the input electromagnetic field. Moreover, the double directional coupler also preserves the input wave polarization, because it internally has a square waveguide. Separation of orthogonal field components is performed with the combination of a $90^{\circ}$ polarizer and an ortho-mode transducer.

The polarizer connected to the OMT, is a differential $90^{\circ}$ phase shifter for the two orthogonal propagation modes $\left(\mathrm{TE}_{10}\right.$ and $\left.\mathrm{TE}_{01}\right)$ in the square waveguide. The polarizer is rotated $45^{\circ}$ in relation to the $x$-axis of the OMT. The constant phase difference between the two modes is achieved by internal stepped ridges in the square waveguide, placed in its four internal walls. When the electric field at the polarizer input has a linear horizontal or vertical orientation (see Figure A1), each orthogonal component propagates along the ridged square waveguide with a different insertion phase. At the polarizer output the two components have a $90^{\circ}$ phase difference between them.

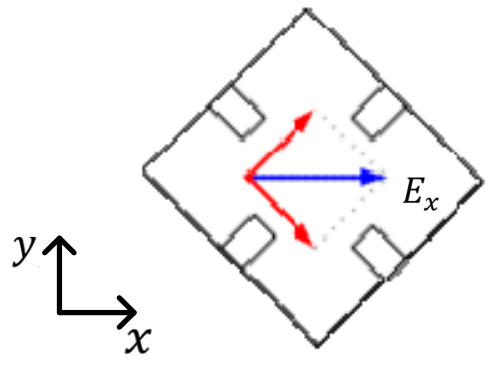

(a)

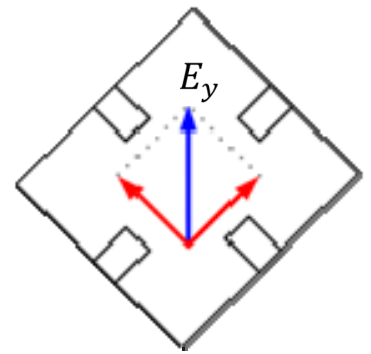

(b)

Figure A1. Polarizer input. (a) Horizontal $\left(E_{x}\right)$ and (b) vertical ( $\left.E_{y}\right)$ electric field (blue). With orthogonal $\mathrm{TE}_{10}$ and $\mathrm{TE}_{01}$ components (red).

The OMT is based on a turnstile junction with an inserted scatterer [38]. It is designed with a circular waveguide input and two rectangular waveguide in-phase outputs. The assembly polarizer-OMT (see Figure A2) has three physical ports and four electrical ports, since at the polarizer input there are two orthogonal modes $\left(\mathrm{TE}_{10}\right.$ and $\left.\mathrm{TE}_{01}\right)$. 


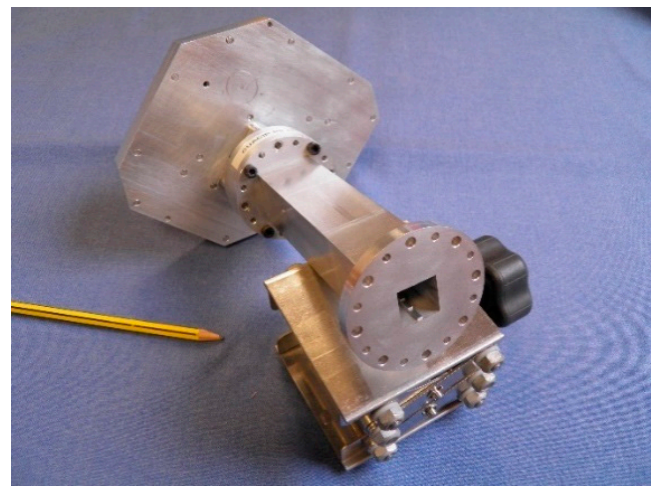

(a)

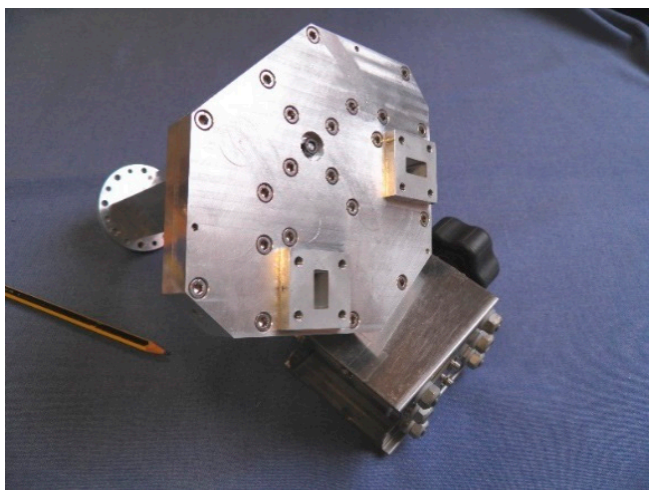

(b)

Figure A2. Assembly polarizer-OMT: (a) polarizer square waveguide input view; (b) OMT rectangular waveguide outputs view.

The four electrical ports for the S-parameters matrix of the polarizer-OMT assembly are: port \#1: OMT output $v_{x}$; port \#2: OMT output $v_{y}$; port \#3: polarizer input $E_{x}$; and port \#4: polarizer input $E_{y}$, as it is shown in Figure A3. Scheme with the four electrical ports of the polarizer-OMT assembly.

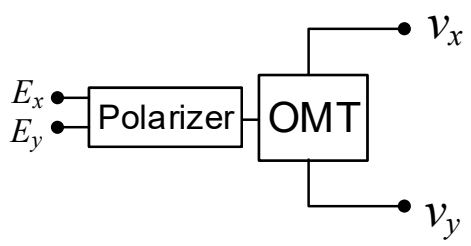

Figure A3. Scheme with the four electrical ports of the polarizer-OMT assembly.

The ideal S-parameters matrix of the polarizer-OMT assembly is:

$$
[S]=\frac{1}{\sqrt{2}}\left[\begin{array}{cccc}
0 & 0 & 1 & -j \\
0 & 0 & -j & 1 \\
1 & -j & 0 & 0 \\
-j & 1 & 0 & 0
\end{array}\right]
$$

A reference phase $\left(0^{\circ}\right)$ is taken for the transmission parameter relating the polarizer input $E_{x}$ with the OMT output $v_{x}$. According to Equation (A1), with an input horizontal electric field $E_{x}$ coming into port \#3, the two OMT output signals have the same amplitude but are $90^{\circ}$ phase shifted between them. Besides, the same happens when an input vertical electric field $E_{y}$ comes into port \#4, providing the OMT output signals with same amplitude and a $90^{\circ}$ phase difference.

In general, for a total electric field, received by the feedhorn antenna, with horizontal and vertical components, as it is expressed in Equation (2) the output voltages from the OMT can be calculated using the above S-parameter matrix. The results are in Equations (3) and (4).

\section{Appendix B}

The correlator module in the BEM combines signals from both branches to obtain additions or subtractions of microwave signals with, or without, a fixed $90^{\circ}$ phase shift. Correlator circuits are implemented in microstrip technology in two sub-bands (10-14 GHz and 16-20 GHz). Each correlation module is a six-port network, and its schematic is outlined in Figure A4. The correlator inputs $v_{i n x}$ and $v_{\text {iny }}$ are the OMT outputs $v_{x}$ and $v_{y}$ amplified, phase shifted and split into sub-bands through the two different branches. The input voltages are combined in the correlator providing four output voltages proportional 
to a combination of the three Stokes parameters (I, Q and $U$ ) of an incoming wave. The combination is carried out using two power splitters, two $3 \mathrm{~dB} / 90^{\circ}$ hybrid couplers and a broadband $90^{\circ}$ phase shifter in one branch. Scattering matrix of an ideal correlator without unbalances is given by:

$$
S_{c o r r}=\frac{-1}{2}\left[\begin{array}{cc}
j & 1 \\
1 & j \\
j & -j \\
1 & 1
\end{array}\right]
$$

For ideal subsystems and unity gain amplifiers, the correlator inputs are $v_{\text {inx }}=v_{x}$ and $v_{i n y}=v_{y} e^{j \phi}$, where $\phi$ is the phase shift between branches in the BEM. Subsequently, the output voltages are computed as

$$
\left[\begin{array}{l}
v_{1} \\
v_{2} \\
v_{3} \\
v_{4}
\end{array}\right]=S_{c o r r}\left[\begin{array}{c}
v_{x} \\
v_{y} e^{j \phi}
\end{array}\right]
$$

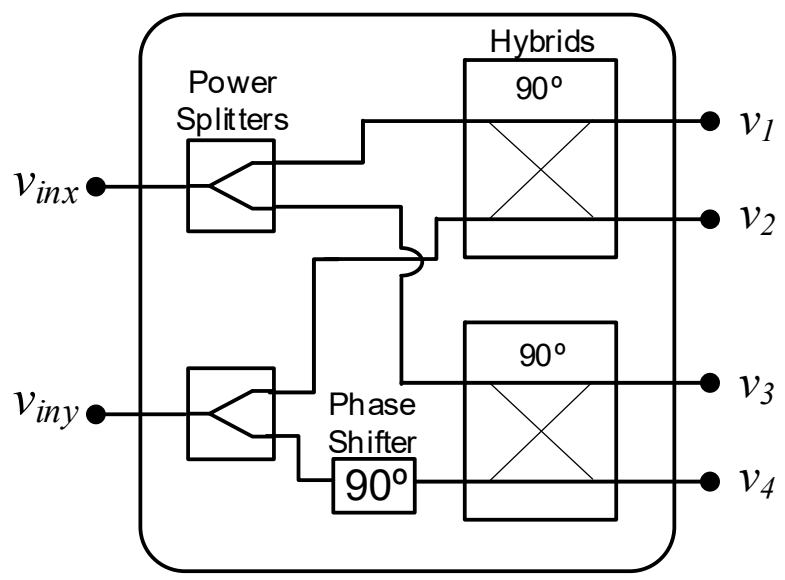

Figure A4. Schematic of the correlation module.

\section{References}

1. Zhu, L.; Suomalainen, J.; Liu, J.; Hyyppä, J.; Kaartinen, H.; Haggren, H. A Review: Remote Sensing Sensors. Multi-purposeful Application of Geospatial Data; IntechOpen: London, UK, 2017; Chapter 2; ISBN 978-1-78923-109-0. Available online: https: //www.intechopen.com/books/multi-purposeful-application-of-geospatial-data/a-review-remote-sensing-sensors (accessed on 1 December 2017). [CrossRef]

2. Ulaby, F.T.; Long, D.G. Microwave Radar and Radiometric Remote Sensing; The University of Michigan Press: Ann Arbor, MI, USA, 2014; ISBN 978-0-472-11935-6.

3. Srivastava, H.; Patel, P.; Sharma, Y.; Navalgund, R. Large-Area Soil Moisture Estimation Using Multi-Incidence-Angle RADARSAT1 SAR Data. IEEE Trans. Geosci. Remote Sens. 2009, 47, 2528-2535. [CrossRef]

4. Paloscia, S.; Pettinato, S.; Santi, E.; Notarnicola, C.; Pasolli, L.; Reppucci, A. Soil Moisture Mapping using Sentinel-1 Images: Algorithm and Preliminary Validation. Remote Sens. Environ. 2013, 134, 234-248. [CrossRef]

5. Gorrab, A.; Zribi, M.; Baghdadi, N.; Mougenot, B.; Fanise, P.; Chabaane, Z. Retrieval of Both Soil Moisture and Texture Using TerraSAR-X Images. Remote Sens. 2015, 7, 10098-10116. [CrossRef]

6. Naeimi, V.; Scipal, K.; Bartalis, Z.; Hasenauer, S.; Wagner, W. An Improved Soil Moisture Retrieval Algorithm for ERS and METOP Scatterometer Observations. IEEE Trans. Geosci. Remote Sens. 2009, 47, 1999-2013. [CrossRef]

7. Jackson, R.D. Soil Moisture Inferences from Thermal-Infrared Measurements of Vegetation Temperatures. IEEE Trans. Geosci. Remote Sens. 1982, 20, 282-286. [CrossRef]

8. Kidd, C.; Matsui, T.; Ringerud, S. Precipitation Retrievals from Passive Microwave Cross-Track Sensors: The Precipitation Retrieval and Profiling Scheme. Remote Sens. 2021, 13, 947. [CrossRef]

9. Koch, P.M.; Kesteven, M.; Nishioka, H.; Jiang, H.; Lin, K.Y.; Umetsu, K.; Romeo, B. The AMiBA Hexapod Telescope Mount. Astrophys. J. 2009, 694, 1670. [CrossRef] 
10. Simon, S.M.; Appel, J.W.; Campusano, L.E.; Choi, S.K.; Crowley, K.T.; Essinger-Hileman, T.; Gallardo, P.; Ho, S.P.; Kusaka, A.; Nati, F.; et al. Characterizing Atacama B-mode Search Detectors with a Half-Wave Plate. J. Low Temp. Phys. 2016, 184, 534-539. [CrossRef]

11. Rubiño-Martin, J.A.; Rebolo, R.; Aguiar, M.; Génova-Santos, R.; Gómez-Reñasco, F.; Herreros, J.M.; Hoyland, R.J.; LópezCaraballo, C.; Santos, A.E.P.; De La Rosa, V.S.; et al. The QUIJOTE-CMB experiment: Studying the polarisation of the galactic and cosmological microwave emissions. In Proceedings of the SPIE, Ground-Based and Airborne Telescopes IV. Int. Society for Optics and Photonics, Amsterdam, The Netherlands, 1 July 2012; Volume 8444. [CrossRef]

12. Cornelison, J.; Ade, P.A.R.A.; Ahmed, Z.; Amiri, M.; Barkats, D.; Thakur, R.B.; Bischoff, C.; Bock, J.; Boenish, H.; Bullock, E.; et al. Polarization Calibration of the BICEP3 CMB polarimeter at the South Pole. In Proceedings of the SPIE, Millimeter, Submillimeter, and Far-Infrared Detectors and Instrumentation for Astronomy X, Online Only, 13 December 2020; Volume 11453. [CrossRef]

13. Kosowsky, A. Cosmic Microwave Background Polarization. Ann. Phys. 1996, 246, 49-85. [CrossRef]

14. Buzzelli, A.; Cabella, P.; Gasperis, G.; Vittorio, N. Polarization of Cosmic Microwave Background. J. Phys. Conf. Ser. 2016, 689, 12003. [CrossRef]

15. Aumont, J.; Macías-Pérez, J.F.; Ritacco, A.; Ponthieu, N.; Mangilli, A. Absolute calibration of the polarisation angle for future CMB B-mode experiments from current and future measurements of the Crab nebula. Astron. Astrophys. 2020, 634, A100. [CrossRef]

16. Gaier, T.C. Coherent radiometers for cosmic microwave background polarization detection. Proc. SPIE 4843 Polarim. Astron. 2003. [CrossRef]

17. Aiken, R.W. Testing Inflationary Cosmology with the BICEP1 and BICEP2 Experiments. Ph.D. Thesis, California Institute of Technology, Pasadena, CA, USA, 2013.

18. The Polarbear Collaboration; Ade, P.A.R.; Aguilar, M.; Akiba, Y.; Arnold, K.; Baccigalupi, C.; Barron, D.; Beck, D.; Bianchini, F.; Boettger, D.; et al. A Measurement of the Cosmic Microwave Background B-mode Polarization Power Spectrum at Sub-degree with POLARBEAR. Astrophys. J. 2014, 794, 171-192. [CrossRef]

19. Padilla, I.L.; Eimer, J.R.; Li, Y.; Addison, G.E.; Ali, A.; Appel, J.W.; Bennett, C.L.; Bustos, R.; Brewer, M.K.; Chan, M.; et al. Two-year Cosmology Large Angular Scale Surveyor (CLASS) Observations: A Measurement of Circular Polarization at $40 \mathrm{GHz}$. arXiv 2019, arXiv:1911.00391. [CrossRef]

20. Choi, S.K.; Hasselfield, M.; Ho, S.-P.P.; Koopman, B.; Lungu, M.; Abitbol, M.H.; Addison, G.E.; Ade, P.A.R.; Aiola, S.; Alonso, D.; et al. The Atacama Cosmology Telescope: A measurement of the Cosmic Microwave Background power spectra at 98 and 150 GHz. J. Cosmol. Astropart. Phys. 2020. [CrossRef]

21. Addamo, G.; Ade, P.A.R.; Baccigalupi, C.; Baldini, A.M.; Battaglia, P.M.; Battistelli, E.S.; Baù, A.; de Bernardis, P.; Bersanelli, M.; Biasotti, M.; et al. The Large Scale Polarization Explorer (LSPE) for CMB Measurements: Performance Forecast. arXiv 2020, arXiv:2008.11049.

22. Collett, E. Field Guide to Polarization; SPIE Press: Bellingham, WA, USA, 2012; ISBN 978-0-8194-5868-1.

23. Spinelli, S.; Fabbian, G.; Tartari, A.; Zannoni, M.; Gervasi, M. A template of atmospheric $\mathrm{O}_{2}$ circularly polarized emission for cosmic microwave background experiments. Mon. Not. R. Astron. Soc. 2011, 414, 3272-3280. [CrossRef]

24. Bischoff, C.; Brizius, A.; Buder, I.; Chinone, Y.; Cleary, K.; Dumoulin, R.N.; Kusaka, A.; Monsalve, R.; Næss, S.; Newburgh, L.B.; et al. The Q/U Imaging Experiment. Astrophys. J. 2013, 768, 9. [CrossRef]

25. Gasiewski, A.J.; Kunkee, D.B. Calibration and applications of polarization-correlating radiometers. IEEE Trans. Microw. Theory Tech. 1993, 41, 767-773. [CrossRef]

26. O'Dell, C.W.; Swetz, D.S.; Timbie, P.T. Calibration of millimeter-wave polarimeters using a thin dielectric sheet. IEEE Trans. Microw. Theory Techn. 2002, 50, 2135-2141. [CrossRef]

27. Lahtinen, J.; Gasiewski, A.J.; Klein, M.; Corbella, I.S. A calibration method for fully polarimetric microwave radiometers. IEEE Trans. Geosci. Remote Sens. 2003, 41, 588-602. [CrossRef]

28. Lahtinen, J.; Hallikainen, M.T. Retardation Plate for the Calibration of a Fully Polarimetric Radiometer: Determination of Characteristics. IEEE J. Sel. Top. Appl. Earth Obs. Remote. Sens. 2017, 10, 3046-3054. [CrossRef]

29. Peng, J.; Ruf, C.S. Calibration Method for Fully Polarimetric Microwave Radiometers Using the Correlated Noise Calibration Standard. IEEE Trans. Geosci. Remote Sens. 2008, 46, 3087-3097. [CrossRef]

30. King, O.G.; Jones, M.E.; Blackhurst, E.J.; Copley, C.; Davis, R.J.; Dickinson, C.; Taylor, A.C. The C-Band All-Sky Survey (C-BASS): Design and implementation of the northern receiver. Mon. Not. R. Astron. Soc. 2014, 438, 2426-2439. [CrossRef]

31. Gentili, G.G.; Lucci, L.; Nesti, R.; Pelosi, G.; Selleri, S. A Novel Design for a Circular Waveguide Directional Coupler. IEEE Trans. Microw. Theory Tech. 2009, 57, 1840-1849. [CrossRef]

32. Yuan, T.; Yin, X.; Zhao, H.; Shi, J.; Zhong, W.; Liu, Q. A design of Q-band noise injector. In Proceedings of the 2014 3rd Asia-Pacific Conference on Antennas and Propagation, Harbin, China, 26-29 July 2014; pp. 1162-1164. [CrossRef]

33. Peverini, O.A.; Tascone, R.; Carretti, E.; Virone, G.; Olivieri, A.; Orta, R.; Monari, J. On-board Calibration System for MillimeterWave Radiometers based on Reference-Polarized Signal Injection. IEEE Trans. Microw. Theory Tech. 2006, 54, 412-420. [CrossRef]

34. Liang, W.; Jia, Z.; Qiu, X.; Hong, J.; Zhang, Q.; Lei, B.; Zhang, F.; Deng, Z.; Wang, A. Polarimetric Calibration of the GaoFen-3 Mission Using Active Radar Calibrators and the Applicable Conditions of System Model for Radar Polarimeters. Remote Sens. 2019, 11, 176. [CrossRef]

35. Aja, B.; Villa, E.; de la Fuente, L.; Artal, E. Double Square Waveguide Directional Coupler for Polarimeter Calibration. IEEE Trans. Microw. Theory Tech. 2019, 67, 1425-1431. [CrossRef] 
36. Aja, B.; Artal, E.; de la Fuente, L.; Villa, E. Acoplador Direccional Doble en Guía Cuadrada. Spanish Patent ES2764742B2, March 2021. (In Spanish)

37. Aja, B.; de la Fuente, L.; Artal, E.; Villa, E.; Cano-de-Diego, J.L.; Mediavilla, A. 10- to 19.5-GHz Microwave Receiver of an Electro-Optical Interferometer for Radio Astronomy. J. Astron. Telesc. Instrum. Syst. 2019, 5, 035007. [CrossRef]

38. Cano, J.L.; Mediavilla, A. Quasi-Octave Bandwidth In-Phase Three-Layer Platelet Orthomode Transducer Using Improved Power Combiners. IEEE Microw. Wirel. Compon. Lett. 2018, 28, 1086-1088. [CrossRef] 